\title{
Prospective relevance of evening carbohydrate and protein intake during adolescence for body composition in young adulthood
}

\author{
T. Diederichs, I. Remmel, S. Rossbach, K. Bolzenius, U. Alexy and A. E. Buyken \\ Institute of Nutritional and Food Sciences - Nutritional Epidemiology, University of Bonn, DONALD Study, \\ Dortmund, Germany
}

Popular weight loss diets commonly advocate the reduction of carbohydrate intake (CHO) in evening meals and/or a high evening protein intake $(\mathrm{PRO})^{(1,2)}$. However, little is known about the longer-term relevance of circadian eating pattern during adolescence, a potential critical period for later body composition ${ }^{(3)}$. Therefore, the aim of the present study was to investigate the prospective relation of evening $\mathrm{CHO}$ and $\mathrm{PRO}$ intake during adolescence to body composition in young adulthood. For comparative purposes, the relevance of morning and daily $\mathrm{CHO}$ and PRO intake was also assessed.

Multivariable regression analyses were performed on data from 182 female and 164 male participants of the DONALD-study with at least two 3-day weighed dietary records during adolescence (baseline: girls 9-14 years; boys 10-15 years) and anthropometric measurements in young adulthood (18-25 years) ${ }^{(4)}$. Fat free mass index $\left(\mathrm{FFMI}, \mathrm{kg} / \mathrm{m}^{2}\right)$ and fat mass index $\left(\mathrm{FMI}, \mathrm{kg} / \mathrm{m}^{2}\right) \mathrm{were}$ estimated from four skinfolds. Day-time specific data was used to calculate morning (before 11 a.m.) and evening (after 6 p.m.) as well as daily $\mathrm{CHO}$ and PRO intake (in \% of energy). In addition, the ratio of carbohydrate to protein (CHO:PRO) was also determined.

Females with a higher evening CHO:PRO ratio during adolescence had lower adult FFMI values $(p=0.018$, see table). Similar trends for lower adult FFMI values were observed for females with a higher evening CHO intake or a lower evening PRO intake during adolescence $(\mathrm{p}<0 \cdot 1$, see table). Evening $\mathrm{CHO}$ or PRO intake was not related to adult FMI among females ( $p>0 \cdot 2)$. Among males, prospective associations were confined to a trend for higher adult FMI levels among those who had consumed more evening CHO during adolescence $(p=0.053)$. Morning CHO or PRO intake was not related to adult body composition among females and males. Analyses of the daily intake revealed that a higher CHO:PRO ratio, a higher CHO intake and a lower PRO intake was again related to lower adult FFMI values among females only (see table).

\begin{tabular}{|c|c|c|c|c|c|c|c|}
\hline \multirow[b]{3}{*}{ Predicting variable } & \multicolumn{6}{|c|}{ Mean adult FFMI in $\mathrm{kg} / \mathrm{m}^{2}(95 \%-\mathrm{CI})^{*}$ among female participants by tertiles $(\mathrm{T})$ of the predicting variable } & \multirow[b]{3}{*}{$\mathrm{p}$ for trend } \\
\hline & \multicolumn{2}{|l|}{$\mathrm{T} 1$} & \multicolumn{2}{|l|}{$\mathrm{T} 2$} & \multicolumn{2}{|l|}{$\mathrm{T} 3$} & \\
\hline & mean & $(95 \%-\mathrm{CI})$ & mean & $(95 \%-C I)$ & mean & $(95 \%-C I)$ & \\
\hline \multicolumn{8}{|l|}{ Evening intake } \\
\hline CHO: PRO ratio & $15 \cdot 64$ & $(15 \cdot 38-15 \cdot 91)$ & $15 \cdot 61$ & $(15 \cdot 35-15 \cdot 88)$ & $15 \cdot 29$ & $(15 \cdot 04-15 \cdot 55)$ & $0.018^{\circ}$ \\
\hline $\mathrm{CHO}$ & $15 \cdot 62$ & $(15 \cdot 36-15 \cdot 90)$ & $15 \cdot 66$ & $(15 \cdot 40-15.93)$ & $15 \cdot 26$ & $(15 \cdot 01-15 \cdot 52)$ & 0.090 \\
\hline PRO & $15 \cdot 31$ & $(15 \cdot 06-15 \cdot 57)$ & $15 \cdot 71$ & $(15.44-15.98)$ & $15 \cdot 52$ & $(15 \cdot 27-15 \cdot 79)$ & $0 \cdot 051$ \\
\hline \multicolumn{8}{|l|}{ Daily intake } \\
\hline CHO:PRO ratio & $15 \cdot 82$ & $(15 \cdot 54-16 \cdot 10)$ & $15 \cdot 56$ & $(15 \cdot 29-15 \cdot 83)$ & $15 \cdot 29$ & $(15 \cdot 03-15 \cdot 56)$ & $0.002^{\circ}$ \\
\hline $\mathrm{CHO}$ & $15 \cdot 91$ & $(15 \cdot 63-16 \cdot 19)$ & $15 \cdot 36$ & $(15 \cdot 10-15 \cdot 63)$ & $15 \cdot 41$ & $(15 \cdot 15-15 \cdot 67)$ & $0.005^{\circ}$ \\
\hline PRO & $15 \cdot 38$ & $(15 \cdot 11-15 \cdot 65)$ & $15 \cdot 54$ & $(15 \cdot 28-15 \cdot 82)$ & $15 \cdot 74$ & $(15.47-16.03)$ & $0.012^{\circ}$ \\
\hline
\end{tabular}

${ }^{*}$ adjusted for FFMI at the beginning of adolescence; ${ }^{\circ}$ mean FFMI values of tertiles were significantly different: $\mathrm{p}<0 \cdot 05$; FFMI - fat free mass index $\left(\mathrm{kg} / \mathrm{m}^{2}\right.$ )

In conclusion, our analyses support a longer-term benefit of a lower evening CHO:PRO intake ratio during adolescence, which was however confined to a higher adult fat free mass among females. Similar, albeit more pronounced associations were observed for the daily CHO:PRO ratio, which argues against a unique relevance of evening $\mathrm{CHO}$ or PRO intake.

1. Pape D, Schwarz R, Trunz-Carlisi E et al. (2006) Schlank im Schlaf (Lean in Sleep). München: Graeve and Unzer publisher.

2. Noakes M \& Clifton P (2005) The CISRO Total Wellbeing Diet. London: Pinguin Books Ltd.

3. Rogol AD, Roemmich JN, Clark PA (2002) Growth at puberty. J Adolesc Health 31, 192-200.

4. Kroke A, Manz F, Kersting M et al. (2004) The DONALD study. History, current status and future perspectives. Eur J Nutr 43, 45-54. 